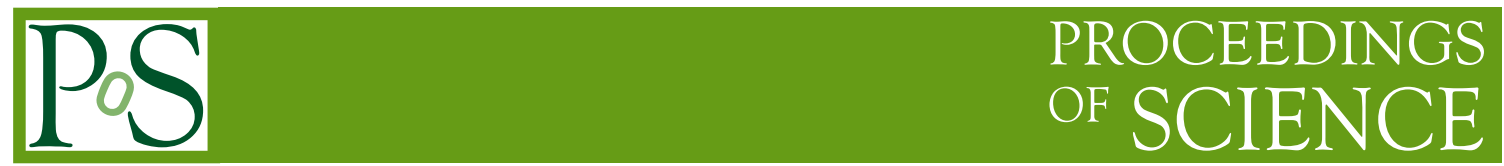

\title{
Parameters in a Walecka-type model for the deuteron
}

\section{Bernard BAKKER*}

Vrije Universiteit, Amsterdam, The Netherlands

E-mail: blg.bakkerafew.vu.nl

\section{Elmar Biernat}

Institute for Physics, Karl-Franzens Universität Graz,

Universitätsplatz 5, A-8010 Graz, Austria

E-mail: elmar.biernat@uni-graz.at

We put forward a proposal for a benchmark calculation of the deuteron form factors. The purpose of the benchmark is to define more accurately what we mean by 'relativistic effects'. A first step to that goal is made here: We define a model Lagrangean in the spirit of the Walecka model, namely one having only a scalar meson $\sigma$, and a vector one, $\omega$. In order to regularize this model, two Pauli-Villars particles are introduced, one regularizing the scalar exchange and one regularizing the vector exchange.

Light Cone 2010: Relativistic Hadronic and Particle Physics June 14-18, 2010

Valencia, Spain

\footnotetext{
* Speaker.
} 


\section{Introduction}

If one studies the literature on treatments of nuclear systems 'including relativity' with ones that are called 'non-relativistic', one notices that there is no consensus about what these terms mean. Therefore, one may try to find some common ground where all researchers in few-body systems can agree.

The present note is a first step in this direction. We define a Lagrangean that is simple enough to derive a simple, effective nucleon-nucleon interaction, but realistic enough in the sense that this interaction supports an S-wave bound state that closely resembles the S-wave part of the deuteron. The Lagrangean chosen is inspired by the $\sigma-\omega$-model, also known as the Walecka model [1]. The present model means a drastic simplification, which of course does not do justice to the sophistication of realistic nucleon-nucleon interaction, found to be needed to achieve precise agreement with the data.

In view of the necessity of regularization and the fact that in different relativistic approaches regularization may take different forms, part of the model proposed here is Pauli-Villars regularization [2]. This amounts to introducing for every exchanged physical boson an unphysical one that regulates the boson propagator in such a way that the interaction defined by boson exchange becomes well-behaved in momentum space.

Using this regulated Lagrangean and the boson-exchange idea to determine the effective interaction between two nucleons, one can now use this interaction in many 'relativistic' or 'nonrelativistic' formalisms. Below we apply this idea in the simplest possible context, namely to write the interaction in the form of a static potential to be used in the non-relativistic Schrödinger equation. Our model is compared throughout to the Malfiet-Tjon potential [3].

In the benchmark proposed here, nucleon-nucleon interactions based on effective-field theory are not included, solely for the reason that they are limited to low energies by construction.

\section{Lagrangean density of the Walecka model}

The interaction Lagrangean densities in the Walecka model are given by $[1,4,5]$

$$
\mathscr{L}_{\mathrm{NN} \sigma}=g_{\sigma} \bar{\psi} \psi \sigma, \quad \mathscr{L}_{\mathrm{NN} \omega}=i g_{\omega} \bar{\psi} \gamma_{\mu} \psi \omega^{\mu}+\frac{f_{\omega}}{4 m_{\mathrm{N}}} \bar{\psi} \sigma_{\mu \nu} \psi\left(\partial^{\mu} \omega^{v}-\partial^{v} \omega^{\mu}\right)
$$

Here $\psi$ denotes the nucleon field of mass $m_{\mathrm{N}}, \sigma$ denotes a neutral scalar meson field of mass $m_{\sigma}$ and $\omega^{\mu}$ denotes a neutral vector meson field of mass $m_{\omega}$. $\mathscr{L}_{\mathrm{NN} \sigma}$ describes the scalar meson exchange and $\mathscr{L}_{\mathrm{NN} \omega}$ describes the vector meson exchange between the nucleons. For simplicity, we take $f_{\omega}=0$.

Using the Feynman rules the second-order amplitudes for scalar and vector exchange become, respectively

$$
\begin{aligned}
& V_{\sigma}^{(2)}=g_{\sigma}^{2} \bar{u}\left(1^{\prime}\right) u(1) \frac{1}{\left(p_{1}^{\prime}-p_{1}\right)^{2}-m_{\sigma}^{2}} \bar{u}\left(2^{\prime}\right) u(2) \\
& V_{\omega}^{(2)}=-g_{\omega}^{2} \bar{u}\left(1^{\prime}\right) \gamma_{\mu} u(1) \frac{g^{\mu \nu}-\left(p_{1}^{\prime \mu}-p_{1}^{\mu}\right)\left(p_{2}^{\prime}-p_{2}^{v}\right) / m_{\omega}^{2}}{\left(p_{1}^{\prime}-p_{1}\right)^{2}-m_{\omega}^{2}} \bar{u}\left(2^{\prime}\right) \gamma_{v} u(2) .
\end{aligned}
$$


Note that for simplicity we take only pure vector exchange for the $\omega$ meson. This can be justified by looking at a comparison of different models for the vector-meson exchange in Erkelenz's paper[2]. Realistic models favour no, or only very small tensor coupling for the $\omega$ meson.

For the Pauli-Villars particles, the same basic forms for the second-order matrix elements are used with one difference: The amplitudes have opposite sign. This sign difference is of course the reason why the Pauli-Villars bosons provide regularization of the potentials.

\section{Static potentials}

In the static and non-relativistic approximation, defined here by replacing the Dirac spinors by their large components, taking the nucleons on-mass-shell, and replacing their energies in the Dirac spinors by their masses, the amplitudes turn out to be

$$
\tilde{V}_{\sigma}(\boldsymbol{q})=-\frac{g_{\sigma}^{2}}{\boldsymbol{q}^{2}+m_{\sigma}^{2}}, \quad \tilde{V}_{\omega}(\boldsymbol{q})=\frac{g_{\omega}^{2}}{\boldsymbol{q}^{2}+m_{\omega}^{2}} .
$$

We used the Dirac equations for the spinors to remove the trensor term in the vector-meson propagator Eq. (2.2):

$$
\bar{u}\left(\boldsymbol{p}^{\prime}\right) \gamma_{\mu}\left(p^{\prime}-p\right)^{\mu} u(\boldsymbol{p})=\bar{u}\left(\boldsymbol{p}^{\prime}\right)\left(m_{\mathrm{N}}-m_{\mathrm{N}}\right) u(\boldsymbol{p})=0,
$$

The potentials in configuration space are given by the Fourier transforms of the expressions Eq. (3.1):

$$
V_{\sigma}(r)=-\frac{g_{\sigma}^{2}}{4 \pi} \frac{\mathrm{e}^{-m_{\sigma} r}}{r}, \quad V_{\omega}(r)=\frac{g_{\omega}^{2}}{4 \pi} \frac{\mathrm{e}^{-m_{\omega} r}}{r}
$$

where $r=|\boldsymbol{r}|$.

\section{Pauli-Villars regularization}

In the Pauli-Villars regularization prescription one introduces fictitious heavy particles in the Lagrangean density. Then the regularized interaction Lagrangean density is given by ${ }^{1}$

$$
\mathscr{L}_{\mathrm{int}}=g_{\sigma} \bar{\psi} \psi \sigma+i g_{\omega} \bar{\psi} \gamma_{\mu} \psi \omega^{\mu}+i g_{\sigma} \bar{\psi} \psi \eta+g_{\omega} \bar{\psi} \gamma_{\mu} \psi \theta^{\mu}
$$

where $\eta$ and $\theta^{\mu}$ are the Pauli-Villars fields with large masses $\Lambda_{\sigma}$ and $\Lambda_{\omega}$, respectively. Note that we couple the Pauli-Villars bosons with the same strength as the physical bosons but with an additional factor $i$, which translates in second order into a relative minus sign of the amplitudes.

This introduction of Pauli-Villars fields in the Lagrangean results in making the following replacement for the meson propagators in Eq. (3.1):

$$
\frac{1}{\boldsymbol{q}^{2}+m^{2}} \longrightarrow \frac{1}{\boldsymbol{q}^{2}+m^{2}}-\frac{1}{\boldsymbol{q}^{2}+\Lambda^{2}}
$$

\footnotetext{
${ }^{1}$ Note that we include only one Pauli-Villars field for either meson. This is sufficient for cutting off the kernels in momentum space if the effective interaction is obtained by taking matrix elements between on-mass-shell nucleons. Otherwise, e.g. if one uses the Bethe-Salpeter equation, more Pauli-Villars fields are needed[7]
} 
Table 1: Values for the masses and coupling constants. They are chosen such that the deuteron binding energy $E_{B}$ and the scattering length $a_{t}$ are well reproduced. These values are similar to those proposed in [6].

\begin{tabular}{|l|r|r|r|}
\hline Parameter (units) & present model & present model, no PV & Malfliet-Tjon model [3] \\
\hline$E_{B}(\mathrm{MeV})$ & -2.224575 & -3.36772 & -2.27203 \\
\hline$a_{t}(\mathrm{fm})$ & 5.4151 & 4.58658 & 5.4739 \\
\hline$g_{\sigma}^{2} / 4 \pi$ & 6.31 & 6.31 & 3.22749 \\
\hline$g_{\omega}^{2} / 4 \pi$ & 18.617 & 18.617 & 7.40758 \\
\hline$m_{\sigma}\left(\mathrm{MeV} / \mathrm{c}^{2}\right)$ & 400 & 400 & 306.8 \\
\hline$m_{\omega}\left(\mathrm{MeV} / \mathrm{c}^{2}\right)$ & 782.7 & 782.7 & 613.6 \\
\hline$\Lambda_{\sigma}\left(\mathrm{MeV} / \mathrm{c}^{2}\right)$ & 1000 & $\infty$ & $\infty$ \\
\hline$\left(\Lambda_{\omega}\left(\mathrm{MeV} / \mathrm{c}^{2}\right)\right.$ & 1500 & $\infty$ & $\infty$ \\
\hline
\end{tabular}

\section{Solving the Schrödinger equation and fixing the parameters}

The two-body S-wave Schrödinger equation for the reduced wave function $u_{0}(r)=r \psi_{0}(r)$, subject to the boundary condition $u_{0}(0)=0$,

$$
\left[-\frac{\hbar^{2}}{2 m_{\mathrm{red}}} \frac{\mathrm{d}^{2}}{\mathrm{~d} r^{2}}+V(r)\right] u(r)=E u(r), \quad \text { with } \quad m_{\mathrm{red}}=\frac{m_{p} m_{n}}{m_{p}+m_{n}}=938.92 \mathrm{MeV} / c^{2} \approx m_{\mathrm{N}},
$$

is solved numerically.

We use the existing data on the recommended $\sigma$-meson mass, the experimental $\omega$-meson mass, the deuteron binding energy, and the triplet scattering length to fix the parameters. For the Pauli-Villars particles we use the mass values (cut off values) $\Lambda_{\sigma}=1000 \mathrm{MeV} / c^{2}$ and $\Lambda_{\omega}=$ $1500 \mathrm{MeV} / c^{2}$, which are reasonable values for these cut offs, although they are somewhat smaller than usual. We did not vary them, because the model proposed here is simply not realistic enough to warrant much effort in this direction. Yet, we wanted this model to produce a reasonable S-wave deuteron wave function. The values of the parameters we found are given in Table 1.

The potential is depicted in Fig. 1 together with the simple Malfliet-Tjon potential MT III [3]. It is clear that the present model has stronger attraction at intermediate range and stronger repulsion at short range than the Malfliet-Tjon potential. This difference can be expected to show up in the bound-state wave function at intermediate range.

As a characteristic quantity that measures the quality of the wave function nonlocally, we calculated the mean square radius defined by [8]

$$
\left\langle r^{2}\right\rangle:=\frac{1}{4} \int_{0}^{\infty} \mathrm{d} r r^{2} u_{0}^{2}(r)
$$

We find a value for the root-mean-squared radius $\sqrt{\left\langle r^{2}\right\rangle}=1.95015 \mathrm{fm}$ close to Malfliet-Tjon's value $\sqrt{\left\langle r^{2}\right\rangle}=1.97625 \mathrm{fm}$. This is also in accordance with other results for the deuteron root mean square radius [8]. The triplet scattering length $a_{t}$ is obtained from the solution $u_{0}(r)$ of the Schrödinger equation Eq. (5.1) corresponding to $E=0$, see Fig. 2 . We find the value $a_{t}=5.421 \mathrm{fm}$, compared to the experimental value of $5.432 \mathrm{fm}[9]$.

We checked the importance of the Pauli-Villars bosons in the static approximation, where they are not needed for convergence. In Table 1 we give the values of the energy and the scattering 

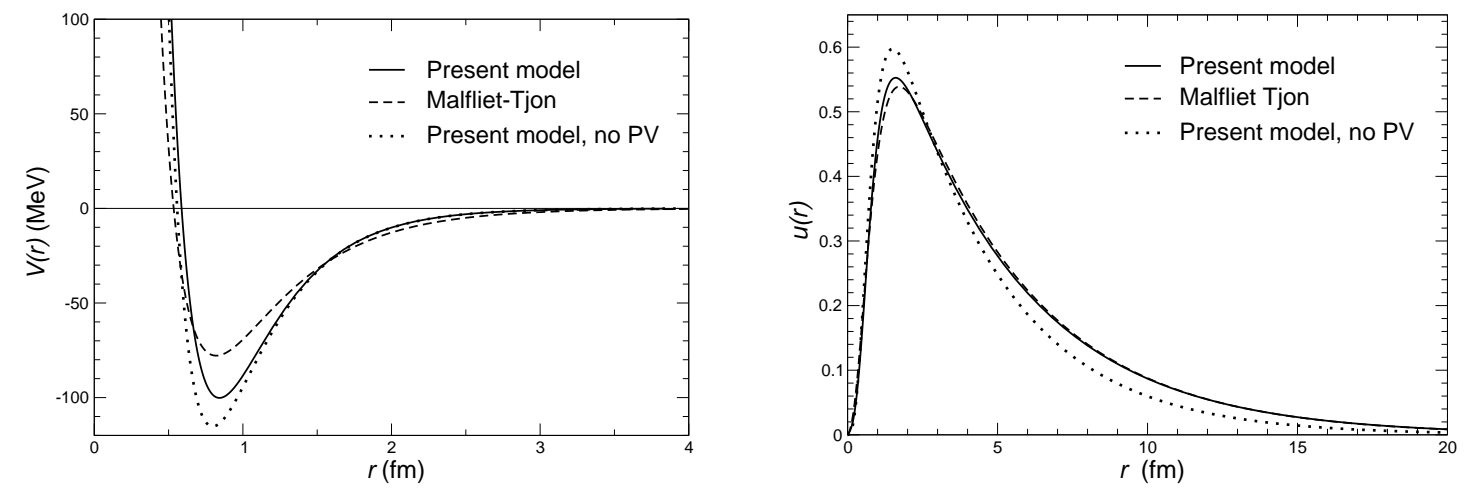

Figure 1: The present potential and wave functions with and without Pauli-Villars regularization compared with the Malfliet-Tjon potential [3]. The parameters are given in Table 1.

length also in the situation that these bosons are omitted. Clearly, the values of the bound-state energy, $-3.368 \mathrm{MeV}$, compared to $-2.2246 \mathrm{MeV}$, and $4.587 \mathrm{fm}$ compared to $5.421 \mathrm{fm}$, are quite reasonable, although of course far outside the error bounds of the experimental data.

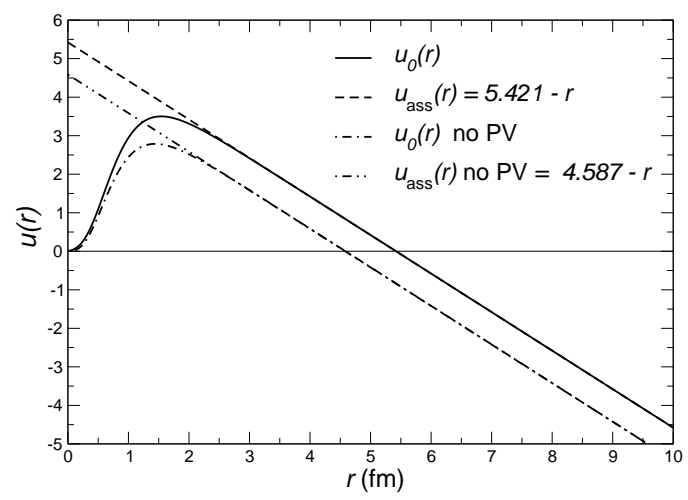

Figure 2: Wave functions at energy $E=0$ for the present model with and without Pauli-Villars regularization included in the potential. The scattering length is determined by a linear fit to the wave function at large $r$.

\section{Form Factor}

As we make no difference between neutrons and protons in our simple benchmark model, we calculate the body form factor of the bound state only. For the S-wave bound state found here, it is given in terms of the normalized wave function by the well-known formula

$$
F(q)=\int_{0}^{\infty} \mathrm{d} r j_{0}(q r) u_{0}^{2}(r)
$$


The normalization of $u_{0}$ guarantees $F(0)=1$. The resulting form factor for the present model is depicted in Fig. 2, which at small $q$ behaves as

$$
F(q) \sim 1-\frac{q^{2}}{6} \int_{0}^{\infty} \mathrm{d} r r^{2} u^{2}(r)=1-4\left\langle r^{2}\right\rangle \frac{q^{2}}{6} .
$$

(Note the factor 4 that comes from the factor $1 / 4$ in the definition of $\left\langle r^{2}\right\rangle$, Eq. (5.2).)
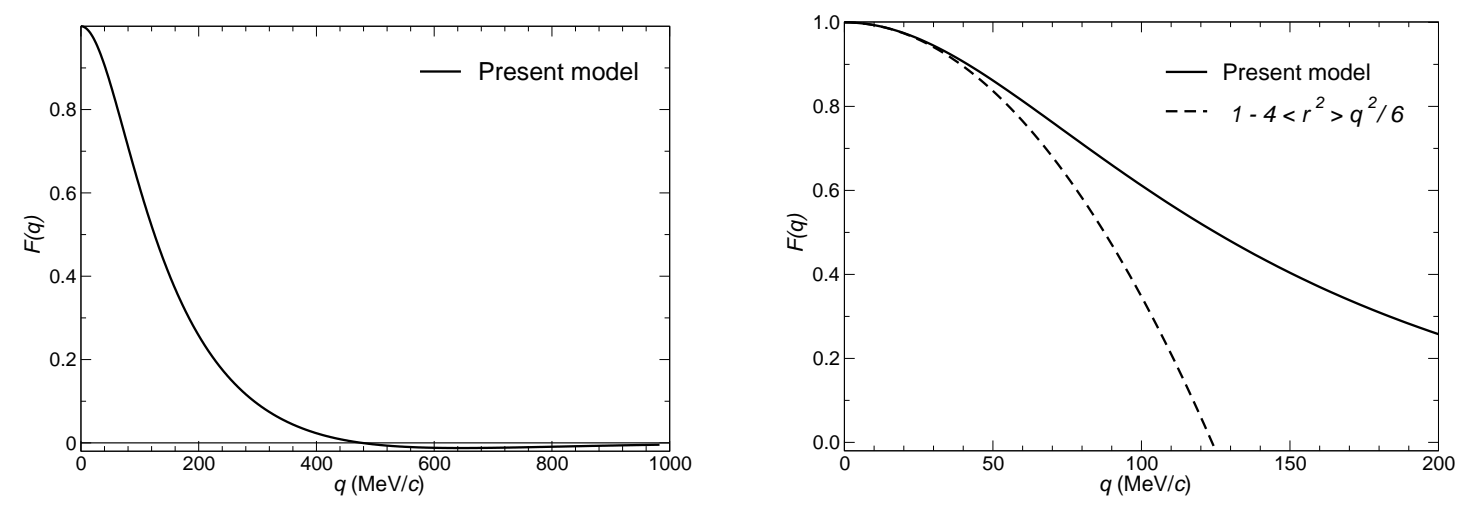

Figure 3: The form factor in the present model including Pauli-Villars regularization. The right-hand panel shows the behaviour at small values of $q^{2}$. The form factor for the Malfliet-Tjon deuteron is indistinguishable on this scale.

\section{Momentum-Space Calculations}

In order to facilitate the comparison of our model with calculations performed in momentum space, we transformed the Schrödinger equation to momentum space and solved it for the energy and the wave function of the bound state. The momentum-space wave function $\omega_{0}(p)$ is shown in Fig. 4. On this scale, we cannot see the difference between the Malfliet-Tjon wave function and the one obtained with the present model. Looking at Fig. 1 we see that in coordinate space the two models differ most below a few fm, so we may expect the momentum-space wave functions to be different at momenta around $200 \mathrm{MeV} / c$, which turns out to be true, as Fig. 4 shows.

For small values of the momenta the momentum-space wave function is dominated by the asymptotic behaviour of the coordinate-space wave function, namely $\psi_{0}(r) \sim a \exp (-\alpha r)$. The corresponding behaviour in momentum space is $\omega_{0}(p) \sim A /\left(\alpha^{2}+p^{2}\right)$. The latter is plotted in Fig. 5.

\section{Relativistic Kinetic Energy}

The simplest modification of the static, non-relativisitic model replaces the kinetic-energy operator $\boldsymbol{p}^{2} / m_{\mathrm{N}}$ by the operator $2 \sqrt{\boldsymbol{p}^{2}+m_{\mathrm{N}}^{2}}-2 m_{\mathrm{N}}$. It is well known that this replacement effectively decreases the kinetic energy and consequently lowers the energy of the bound state(s) if the potential remains the same. This is indeed what we find. The eigenvalue of the Hamiltonian drops to $-2.73414 \mathrm{MeV}$. 

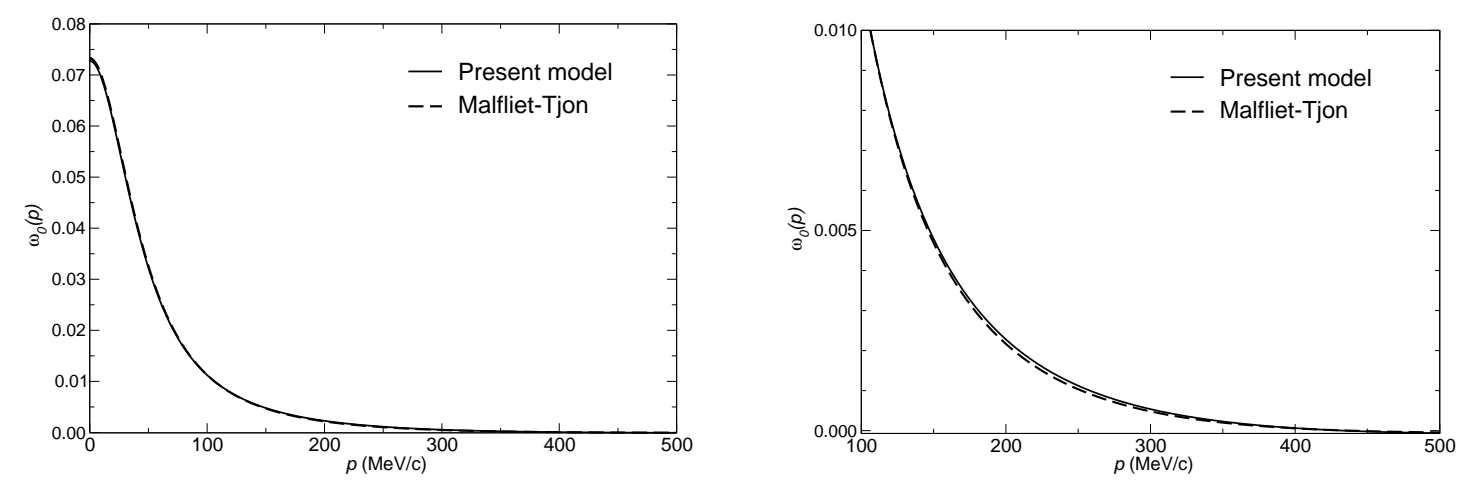

Figure 4: The wave function $\omega_{0}(p)$ in momentum space for the present model including Pauli-Villars regularization. The right-hand panel shows the wave function at small momenta, together with the pole part of the wave function. The wave function for the Malfliet-Tjon deuteron is indistinguishable on this scale.
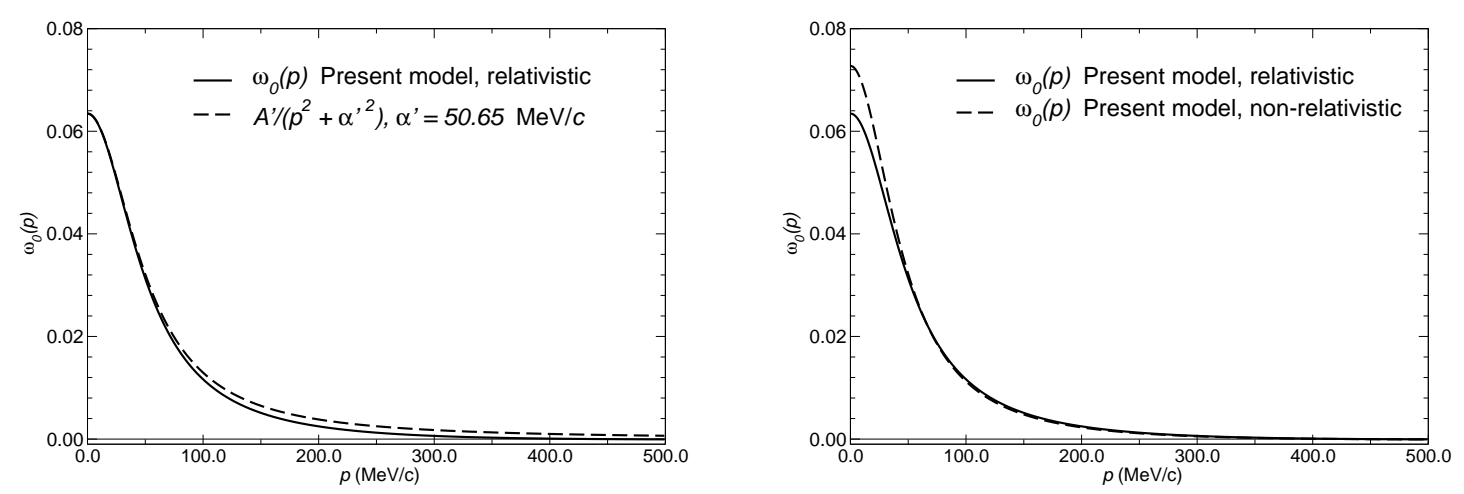

Figure 5: The momentum-space wave function $\omega_{0}(p)$ for the present model including Pauli-Villars regularization and relativistic kinetic-energy operator. Also shown is the pole part. The right-hand panel shows a comparison of the the momentum-space wave functions for the case where the non-relativistic kinetic-energy operator is used and the one where the relativistic operator is used.

In Fig. 6 we show again the form factor for the Walecka-type model, but now together with the one where the relativistic kinetic energy is used. The differences between the two is small, but can be seen most clearly in the semi-log plot.

\section{Summary}

We propose a simple model for a two-nucleon system that is based upon a fully covariant Lagrangean, regulated using Pauli-Villars bosons. In the static approximation the bound-state energy is equal to the deuteron energy and its wave function is close to the deuteron S-wave function found in realistic models. This model can be used to compare non-static interactions, i.e., taking into account the energy dependence of the two-body interaction, and relativistic calculations in different forms of dynamics, based on boson-exchange field-theoretical interactions.

Using this model one may study the bound-state body form factor. Looking at the differences between the form factors obtained using the present Walecka-type model and the Malfliet-Tjon 

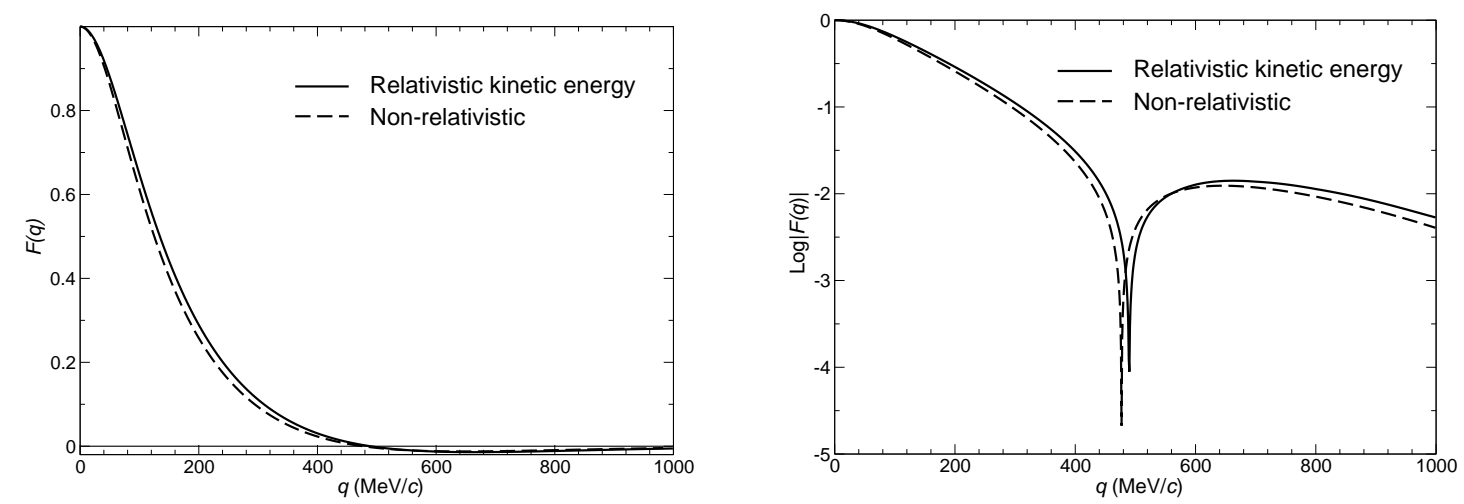

Figure 6: A comparison of the form factors obtained for the present model including Pauli-Villars regularization with the relativistic kinetic-energy operator and the non-relativistic one. The right-hand panel shows the form-factors in semi-log representation

potential, both with non-relativistic kinetic-energy operators, and the same potential combined with the relativistic kinetic energy, we see that the differences are very small.

The simplicity of the model precludes an investigation of deuteron properties that are tied to its D-wave, for instance the quadrupole moment and the quadrupole form factor. Moreover, by limiting the exchange to neutral mesons, some meson-exchange effects do not occur. If it turns out that benchmark calculations employing the present model produce the much desired basis for a precise definition of 'relativistic effects', it will pay off to modify the model in order to make it more realistic, in particular by including pion exchanges.

In relativistic approaches where the nucleons are allowed to go off the mass shell, modifications of the model are necessary.

\section{References}

[1] J.D. Walecka, Ann. Phys. 83, 491 (1974)

[2] W. Pauli and F. Villars, Rev. Mod. Phys. 21, 434 (1949); C. Itzykson and J.-B. Zuber, Quantum Field Theory, (McGraw-Hill, New York, 1980).

[3] R.A. Malfliet and J.A. Tjon, Nucl. Phys. A 127, 161 (1969).

[4] M.M. Nagels, Baryon-Baryon Scattering in a One-Boson-Exchange Potential Model, $\mathrm{PhD}$ thesis, Nijmegen University, (1975).

[5] T.A. Rijken, One-Boson-Exchange Models and Regge Poles, PhD thesis, Nijmegen University (1974).

[6] K. Erkelenz, Phys. Rept. 13, 191 (1974).

[7] V.A. Karmanov, private communication.

[8] R.K. Bhaduri, W. Leidemann, G. Orlandini, and E.L. Tomusiak, Phys. Rev. C 42, 1867 (1990).

[9] T.A. Rijken, Phys. Rev. C 73, 044007 (2006). 